\title{
Experiencias de cuidado institucional: construyendo significados desde la mirada de los niños y las niñas
}

\author{
Experiences of institutional care: \\ Building meanings from children's perspective
}

\author{
Ana Milena Franco Rueda ${ }^{\circ}$
}

Para citar este artículo: Franco, A. M. (2019). Experiencias de cuidado institucional: construyendo significados desde la mirada de los niños y las niñas. Infancias Imágenes, 18(2). [306-315]

\section{Resumen}

Este artículo busca aproximarse a la experiencia de
Recibido: 18-05-2018 - Aceptado: 23-07-2019

\begin{abstract}
This study intends to observe the experiences of children who are under institutional care and to present some observations about the inadequacy of institutional contexts with respect to child protection. The outcome of a dialogue and observation exercise is presented. The exercise was conducted with a group of children under institutional care in Bogotá. In this exercise the constructed meanings around the protection process and the children's institutional stay were explored. Finally, some observations on the importance of prioritising interventions that promote family care and minimise adverse experiences in cases where the transition through institutional care environments is inevitable.
\end{abstract}

Keywords: child welfare, child protection, foster care, social programmes.

1 Docente en Corporación Universitaria Minuto de Dios. Magíster en Psicología y Psicóloga de la Universidad Nacional de Colombia y Licenciada en Ciencias Religiosas de la Pontificia Universidad Javeriana. Correo electrónico: anamifrarue@gmail.com 


\section{Introducción}

El modelo de cuidado institucional, como alternativa de protección a la infancia, ha sido ampliamente cuestionado (Sherr, Roberts y Gandhi, 2017; Naumova et al., 2019) por cuanto constituye un espacio que excluye a los niños y niñas de múltiples posibilidades de participación social y cultural.

El cuidado institucional con frecuencia supone unas condiciones de deprivación social que tienen efectos nocivos a largo plazo en el desarrollo afectivo, cognitivo y social de los niños y niñas (Nadeem et al., 2017; Perego, Caputi, y Ogliari, 2016).

La mayor parte de las investigaciones realizadas con niños y niñas en cuidado institucional se orientan al análisis del impacto de las experiencias de maltrato o negligencia vividas por ellos y a las lesiones o secuelas psicológicas que de ellas puedan derivarse (González, 2014; Gypen, Vanderfaeillie, Maeyer, Belenger y Holen, 2017; TroIler-Renfree, Nelson, Zeanah y Fox, 2016). Suelen ser minoritarias o estar por completo ausentes, alusiones al impacto del cuidado institucional en la subjetividad, las posibilidades de resiliencia o la comprensión que los niños y niñas construyen de esta experiencia. Los niños y niñas en situación de institucionalización han sido abordados como sujetos experimentales, pero no como agentes capaces de construir y resignificar su experiencia.

El presente trabajo busca aproximarse al fenómeno del cuidado institucional infantil e intenta dar voz a los niños y niñas que han vivido esta experiencia dentro de procesos orientados al restablecimiento de sus derechos. Esto con el fin de explorar los significados construidos en torno a dicha experiencia como camino alternativo para cuestionar la conveniencia del cuidado institucional como parte de los procesos de protección infantil. Se trata, además, de un esfuerzo por reivindicar el lugar de la experiencia de los niños y niñas como actores sociales competentes (Villalta, 2013), en contra de las perspectivas "adultocéntricas" que prevalecen en la investigación en torno a la infancia y la niñez (Hirschfeld, 2002).

Para responder a estos objetivos el presente trabajo se ha estructurado en dos apartados. El primero expone los resultados de un ejercicio de diálogo y observación realizado en el año 2011 con niños y niñas de una institución de protección en la ciudad de Bogotá. En el segundo se presentan algunas reflexiones sobre las dificultades inherentes a las prácticas de cuidado institucional y a las posibles alternativas para priorizar el cuidado familiar, como entorno privilegiado para favorecer el bienestar de los niños y niñas.

\section{El cuidado institucional: miradas, experiencias y significados desde los niños y niñas}

En este apartado se ha buscado reconstruir la experiencia del cuidado institucional desde dos ejes fundamentales: la forma en la que los niños comprenden el proceso de restablecimiento de derechos, que en su caso pasa por la separación del núcleo familiar y que concluye bien sea en el retorno a este o en la incorporación a un nuevo grupo familiar; y el significado de la estadía en la institución, desde lo que esto implica tanto en las dinámicas institucionales de cuidado como en la relación que los niños establecen entre sí y con sus cuidadores.

La metodología escogida para esta aproximación combina algunos elementos de la práctica etnográfica y un diseño de entrevista adaptado a los niños. El uso de la etnografía como herramienta parte de la necesidad de conocer los contextos cotidianos de interacción en los que participan los niños y niñas, así como las categorías y dinámicas que en ellos emergen. Esta opción fue facilitada por el hecho de que la investigadora participó como docente ocasional en la institución y, en consecuencia, era una figura conocida para los niños y los demás miembros de la institución. La observación se realizó desde una perspectiva participante, una o dos veces por semana, en períodos de dos horas en promedio y en horarios y actividades diversas (en la mañana y la noche, entre semana y en fines de semana).

En aras de optimizar el proceso de recolección de información se diseñó un formato de entrevista proyectiva orientada a indagar más a fondo sobre la comprensión de la institucionalización que construyen los niños. Se elaboró una maqueta que representa los espacios más frecuentados por los niños y se les pidió que ayudaran a la investigadora a construir la historia de un niño o niña (variaba 
según el sexo del niño entrevistado) que era recibido en la institución, haciendo énfasis en los motivos por los cuales llega, los aspectos positivos o negativos de la permanencia en la fundación y su destino una vez egresa de ella. Adicionalmente, se pidió a los niños que realizaran dibujos en los que representaran las posibles vivencias antes, durante y después de su permanencia en la institución. Esta actividad fue realizada con niños y niñas entre los 8 y los 10 años, que han estado en la institución por periodos superiores a un año, inferiores a un año e inferiores a seis meses.

En lo que respecta al proceso de restablecimiento de derechos, los niños y niñas se muestran conocedores de algunas instancias como el Instituto Colombiano de Bienestar Familiar (ICBF), el Defensor, los hogares sustitutos y las fundaciones y señalan a la Policía o "la patrulla" como agente que conduce a la institución.

Algunos de los niños mencionaron períodos de estadía en hogares sustitutos y en otras fundaciones. La mayoría de los niños coinciden en señalar, como motivos de ingreso a la institución, situaciones de maltrato físico, negligencias en el cuidado o abandono:

Porque a veces los dejan solos y entonces ella se demora y después se ponen a llorar.

Por maltrato social, nos pegan, que le pegan con correa, palo, eh... alambre, con escoba, con un tubo.

Junto al maltrato se señalan situaciones de vulnerabilidad como el huir del núcleo familiar:

[La trajeron] por estar escapándose de la casa de ella. Porque ella, porque ella le gustaba escaparse y no estar con los papás de ella. (Bogotá, septiembre, 2011)

Durante la estadía en la institución algunos niños reciben visitas de sus familiares, especialmente de las mamás, en horarios claramente determinados. Los niños señalan que estas visitas están condicionadas a su comportamiento y los cuidadores al parecer utilizan ese espacio para reportar a los familiares los accidentes o dificultades vividas con los niños: [su mamá vino a visitarlo] Pero ya no, porque, porque yo me he portado mal... le he pegado a mis compañeros. (Bogotá, septiembre, 2011)

Los niños y niñas que aún mantienen vínculos con sus familias y que han permanecido en la institución por períodos inferiores a un año ven la institucionalización como una etapa transitoria y esperan el pronto retorno a sus núcleos familiares. Quienes han permanecido en la institución por más de un año y no tienen contacto con sus familias biológicas mencionan la posibilidad de ir con padres adoptivos, a otras fundaciones o incluso a la "calle":

[...] se los llevó la policía, a la calle, porque ellos ya eran grandes.

A otra fundación por portarse mal o con una familia o con una familia adoptiva.

Que si a Juliana le gusta esta fundación y si cuando cumpla los 18 años va a pasar a otra fundación, [allí le va a ir] mal, porque hay niños grandes que le peguen a ella. (Bogotá, septiembre, 2011)

Es de notar que los niños señalan su propio comportamiento como la variable que determina las posibilidades de ser acogidos en un nuevo entorno familiar o de permanecer en instituciones de protección.

La situación de institucionalización es aludida por los niños desde imaginarios de reclusión que están vinculados con su comportamiento y no con dinámicas familiares o exteriores:

Está en una Fundación y no puede salir, [...] si ella se porta bien muy pronto irá a salir.

Va a pasar a instituciones más grandes y si él sigue así lo mandan por ahí a la cárcel.

Podría pensarse entonces que la institucionalización no es vista por los niños como una medida de protección a su favor, sino como consecuencia de un comportamiento inadecuado (Carreño, 2017). Esto coincide con el reporte de investigaciones que señalan que los niños maltratados tienden a considerar que su comportamiento ha sido la causa del maltrato y que fue confirmado además en el reporte de los niños participantes en el presente trabajo: 
Que los papás le pegaban, porque no hacía caso.

Contribuir a que los niños comprendan el cuidado institucional como una medida de protección que se opone a toda forma de maltrato puede favorecer la capacidad de resiliencia de los niños y niñas y el desarrollo de posturas críticas y alternativas frente a los ciclos de violencia familiar (Benavidez y Miranda, 2007).

Para los niños es claro que la familia es el entorno de cuidado primario, del que provienen y hacia el cual deben ir en forma preferente:

Después se irá con su mamita, con su papito y con sus hermanitos [...] se va a quedar en su casita, [los otros niños] ellos se van a ir, van a poder ir con su familia y algunos, todos, van a poder regresar con su mamita. (Bogotá, septiembre, 2011)

Los niños que aún tienen contacto con sus familias o que llevan poco tiempo en la institución aluden en sus dibujos y expresiones a sus mamás y hermanos; en menor proporción se mencionan otros familiares como padres o tíos. En su gran mayoría manifiestan el deseo de reintegrarse a sus familias, de ir "con mis propios papás" o con "papitos iguales" (figura 1).

Los niños que han permanecido más tiempo en la institución no suelen dibujar ni mencionar figuras parentales, en cambio, dibujan y nombran a sus cuidadores y compañeros. Ellos mencionan con mayor frecuencia las dinámicas de adopción como rutas de salida de la institución, aunque en

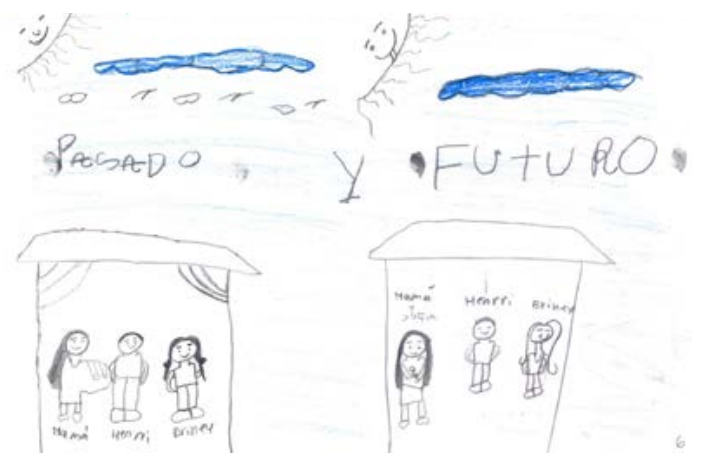

Figura 1. Dibujo realizado por "J" quien espera reintegro al núcleo familiar. general para todos los niños es conocida y cercana esta dinámica:

Se van con otros papás, porque, porque ya le consiguieron papitos nuevos. (Figura 2)

Con respecto a la adopción, los niños señalan que personas adultas que trabajan en la fundación en servicios administrativos son quienes "consiguen" los "papás nuevos" y que esta posibilidad está condicionada a su comportamiento:

Con adopción, con papitos nuevos que uno se va [...] a veces... "Lucía" [nombre ficticio que corresponde en el relato a un adulto que trabaja en asuntos administrativos de la institución]... a veces nos consigue papás porque los otros papás nos maltrataban.

Con papás nuevos [...] es chévere [...]. Portándose uno bien y le llega familia nueva.

En general hay un imaginario favorable a los procesos de adopción, posiblemente construido alrededor de lo que han visto con los compañeros que han sido acogidos en familias adoptivas y que regresan de visita a la institución:

Es chévere, los papás nos cuidan, nos llevan a pasear, nos traen cosas.

Durante su permanencia en la institución los niños se enfrentan por una parte a los ritmos institucionales de cuidado, que se distancian necesariamente de las experiencias familiares; $y$, por otra, a

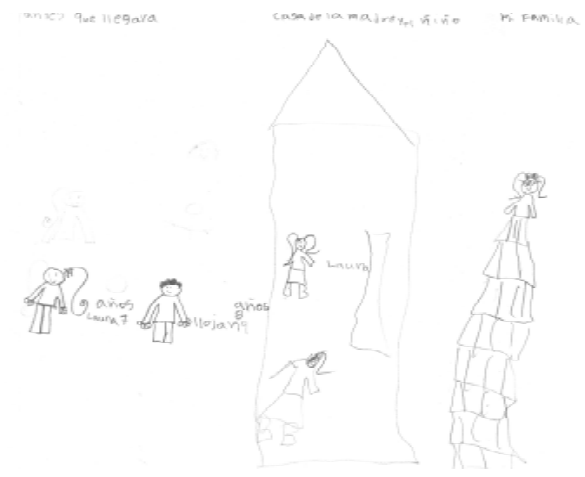

Figura 2. Dibujo realizado por " $A$ " quien espera familia adoptiva. 
la necesidad de interacción y construcción de vínculos con sus compañeros y compañeras, que frecuentemente suelen ser hostiles a quien es nuevo.

La institución, desde la mirada de los niños, es un lugar en donde encuentran "buenas cosas":

Una cama, comida, un juego, un estudio... ropa... Se sentía muy feliz de estar en una casa tan bonita. Es una fundación muy divertida porque tiene parque y todo.

Porque le dan colegio, le... y... le dan comida y le dan actividades, le dan cariño y amor y parque (Bogotá, septiembre, 2011)

Expresiones como estas y alusiones muy frecuentes al parque, la ludoteca y los juguetes hacen pensar que los niños provienen de ambientes de deprivación en los que hay carencia de posibilidades recreativas.

Los adultos significativos que los niños mencionan son primordialmente sus cuidadores, docentes y auxiliares, las psicólogas, la trabajadora social y algunas personas con funciones administrativas.

De los reportes de los niños se deduce la prevalencia de prácticas de cuidado en las que el control de las actividades es predominantemente adulto. Los niños están bajo el cuidado de un personal uniformado, en espacios claramente delimitados. Ellos mismos, durante la semana, permanecen vestidos y peinados de manera uniforme, bajo horarios preestablecidos.

En las dinámicas institucionales la ropa, los juguetes y los espacios son siempre compartidos. Esta realidad, sumada a las experiencias de deprivación vividas por los niños, hace que se desarrollen mecanismos de posesión y apropiación de espacios, como las habitaciones o las camas, y de objetos, como juguetes o utensilios que les han sido obsequiados:

Los amigos no dejan que duerman en sus camas cuando llega nuevo.

J... le reclamó a disgusto que ese no era su cuarto y que por tanto esos no eran sus juguetes.

L... me contó que A... les esculcaba y me mostró lo que guardan en los cajones: una maleta de Hanna Montana "con las cosas para maquillarnos", un trompo de luces que les regalaron en la "fiesta" de la finca a la que fueron, manillas, una crema con un sticker. (Bogotá, septiembre, 2011)

En lo que respecta a las dinámicas de interacción se evidencia que los niños y niñas construyen mecanismos de inclusión y exclusión paralelos al control de los adultos, pero en permanente relación a ellos. Los mecanismos de exclusión se orientan a quien es nuevo o a quien ha establecido alguna relación preferente con las figuras adultas de afecto o autoridad y operan en forma de agresiones físicas, verbales o simbólicas (no darle la mano, no sentarse a su lado, romper los trabajos u objetos de otro, etc.):

Como cuando ellos estaban hablando en secreto y ella no podía hablar con ellos y a ella a veces le tocaba dormirse temprano y a los demás no.

Ha peleado, ha decido groserías, le dicen groserías a él, entonces él comienza a pelear.

Es frecuente que quienes son "víctimas" de agresiones se tornen "victimarios" al ser aceptados dentro de un subgrupo o bien que los "victimarios" se conviertan en "victimas" cuando son excluidos de determinado núcleo. Los líderes suelen ser los niños de mayor edad o tiempo en la institución; la dinámica de liderazgo se reconfigura continuamente en función del "poder" que detente uno u otro niño por su capacidad de reconocimiento frente a los adultos o bien por atributos que los hacen ver invulnerables a los ojos de otros:

A C... no le duele, a él le gusta que le peguen. Si uno sabe pelear no le duele.

Los mecanismos de inclusión y construcción de vínculos giran en torno al compartir juguetes, espacios, actividades, comida, etc. Es frecuente encontrar pequeños grupos que comparten objetos, hacen dibujos o trabajos similares y realizan actividades particulares como entrenamientos de peleas o boxeo, para el caso de los niños, o maquillaje de uñas en el caso de las niñas. Aunque los grupos suelen constituirse por género, el grupo de las niñas más grandes suele acoger o "proteger" a algunos niños más pequeños que entran en conflicto o son excluidos de los grupos masculinos. 


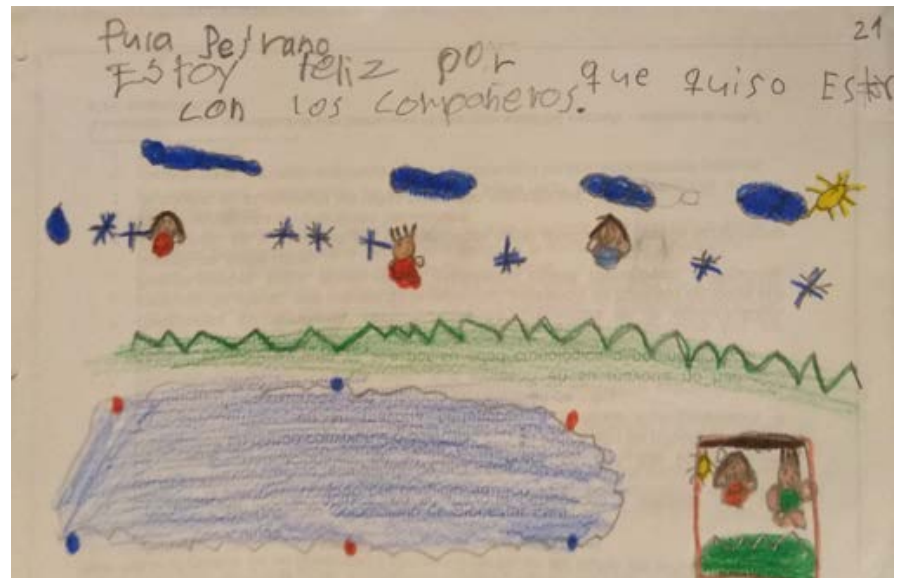

Figura 3. Dibujo sobre experiencias gratificantes dentro de la institución.

\section{¿Institución vs. Familia? ¿ Reestablecer o fragmentar?}

La experiencia de cuidado institucional puede ser comprendida desde la doble dinámica de la ruptura y la reconstrucción de vínculos. La teoría del apego o del vínculo afectivo, propuesta por Bowlby, afirma que "la tendencia a establecer lazos emocionales íntimos con individuos determinados (las figuras de apego) es un componente básico de la naturaleza humana que está presente en el momento mismo del nacimiento y permanece durante toda la vida" (2012, p. 136). Esta necesidad fundamental de establecer lazos emocionales, y el conflicto que se genera en la ruptura de estos, es el eje central para comprender la experiencia del cuidado institucional y para generar estrategias que permitan minimizar su impacto en el desarrollo psicoafectivo de los niños y niñas que la viven. Esta medida, a pesar de ser considerada drástica e indeseable y estar prevista solo como último recurso de protección, continúa siendo muy frecuente en los procesos de restablecimiento de derechos en Colombia (Durán y Valoyes, 2009).

El vínculo primario que se estableció o debió establecerse en el entorno familiar es minado por las experiencias de maltrato y abandono que conducen a los niños a la institucionalización y que se relacionan con secuelas afectivas que se expresan en comportamientos agresivos o de retraimiento (Touza, 2002). Unos y otros manifiestan la dificultad para vincularse con otros que se evidencia en la experiencia de los niños bajo cuidado institucional y que tiende a acentuarse en las dinámicas de relación que favorecen los ritmos institucionales.

La institución que acoge a los niños está en clara ruptura con el entorno familiar y ofrece una nueva forma de cuidado que proviene de figuras adultas, casi exclusivamente femeninas, "uniformadas" en funciones especializadas de educación, cuidado y vigilancia, supletorios de las figuras parentales. En este entorno de protección y control se conjuga la doble mirada que ha caracterizado la concepción moderna de la infancia: por una parte, el niño como sujeto de protección y cuidado y, por otra, como potencial peligro social que es preciso contrarrestar (De la Iglesia y De lorio, 2005).

El entorno institucional está diseñado para restablecer un orden social que ha sido alterado por el maltrato y el abandono, pero también por la inexistencia de vínculos familiares capaces de acoger y nutrir a la infancia. A la base está una acción de beneficencia, moralizante y normalizadora, que se orienta a suplir o paliar las situaciones familiares irregulares que se asocian, al maltrato, el abandono, y en el caso de algunas instituciones, al aborto.

La permanencia en la institución representa una ruptura con los vínculos familiares que paradójicamente se busca restablecer. La institución opera como una estación transitoria que idealmente debe conducir al restablecimiento pleno del vínculo con la familia biológica o a la construcción de nuevos vínculos en el entorno de una familia adoptante. En ese espacio de transición la experiencia subjetiva de sufrimiento reportada por los niños está 
en relación con la ruptura de los lazos familiares y con la exclusión del grupo. Por el contrario, lo que hace satisfactoria la experiencia es la posibilidad de establecer nuevos vínculos emocionales a partir de la amistad y el compartir en actividades lúdicas antes inaccesibles.

El hecho de que el entorno institucional ofrezca ritmos, prácticas de cuidado y figuras adultas muy diversas a las del ambiente familiar puede agudizar la ruptura de los vínculos y dificultar la construcción de nuevos lazos emocionales en el entorno institucional y en los procesos de reintegro o adopción; sobre todo para el caso de los niños que permanecen por períodos prolongados en la institución. Para ellos la salida de la institución a un entorno familiar o a otra institución representa una nueva ruptura, que lesiona una vez más la capacidad de establecer lazos de confianza en las relaciones con otros.

En los dibujos de los niños y niñas prevalece la imagen de una casa. La "casa" puede ser símbolo de un espacio de calidez e intimidad en donde se experimenta seguridad y confianza. Los niños y niñas que viven experiencias de maltrato y cuidado institucional necesitan reconstruir una "casa" que les permita confiar en otros y establecer lazos emocionales perdurables. Las dinámicas de heteronomía y exclusión y los imaginarios de reclusión que los niños construyen sobre su estadía en el entorno institucional son un obstáculo para el proceso de reconstrucción afectiva que debe ser favorecido a través de los mecanismos de restablecimiento de derechos.

\section{Del cuidado institucional a la intervención en perspectiva familliar} Aunque el Código de Infancia y Adolescencia (Ley 1098) establece el derecho de los niños y niñas a tener una familia y no ser separados de ella (artículo 22) y da prevalencia a la ubicación de los niños y niñas en su familia extensa, en los casos en los que resulta necesario retirarlos del cuidado parental (artículo 56), en la práctica, en el proceso de restablecimiento de derechos suele desvirtuarse el lugar de la familia y se recurre a la ubicación en entornos institucionales, sin que hayan sido agotadas todas las posibles opciones de cuidado familiar.
Como señalan Durán y Valoyes (2009), la atención en medio institucional bajo la modalidad de internado está prevista como una medida transitoria en tanto se realiza el reintegro de los niños a sus familias de origen o a familias adoptivas. Las instituciones encargadas de este tipo de atención son de carácter privado, han establecido un contrato con el Estado y están bajo la veeduría del ICBF. Dado este carácter privado se trata de entidades "heterogéneas en sus orígenes, compromisos, recursos, intereses, modalidades de intervención y funcionamiento" (Durán y Valoyes, 2009).

El proceso de restablecimiento de derechos en su conjunto y los procedimientos en los que se opera no prevén la centralidad del papel de la familia en el bienestar y la garantía plena de los derechos de los niños y niñas. En la práctica, las familias que son reportadas al sistema de protección, por supuestas situaciones de maltrato o negligencia, son conminadas a cumplir una serie de requisitos, so pena de que los niños sean retirados del entorno familiar. Sin embargo, estos requerimientos no se acompañan de ningún proceso real de apoyo psicosocial que garantice cambios sostenibles en la dinámica familiar.

Por otra parte, cuando los niños y niñas son ubicados en hogares sustitutos o en instituciones de protección con frecuencia los vínculos con la familia de origen se debilitan a raíz de que las posibilidades de visitas quedan sujetas a la burocracia de los procedimientos legales y, en ocasiones, a las prácticas disciplinarias y de control de las instituciones, en las que las visitas familiares se condicionan al comportamiento de los niños o al cumplimiento de requisitos por parte de los padres. Prácticas institucionales como estas comunican a los niños y a sus familias que las normas de la institución prevalecen sobre los vínculos afectivos de la familia y, en consecuencia, desvirtúan el valor de la pertenencia a un grupo familiar (Minuchin et al., 2000).

Además, la relación que se establece entre las familias y las instituciones de protección o los funcionarios que llevan adelante el proceso es frecuentemente conflictiva y está marcada por la desconfianza y la descalificación mutua. Una buena parte de los profesionales que están a cargo de 
estos procesos desarrollan sobre las familias con las que trabajan, teorías subjetivas en las que se da prevalencia a los aspectos negativos, de déficit y carencia (Gómez y Haz, 2008). La relación de las instituciones con las familias frecuentemente comunica a estas últimas un sentido de negligencia e incapacidad para el cuidado protector de los niños y niñas y la separación prolongada debilita los ya frágiles vínculos familiares y la percepción de autoeficacia de los padres (Minuchin et al., 2000). En oposición a estas prácticas, la investigación ha mostrado que el contacto frecuente con las familias de origen, cuando esto es posible, se relaciona con la disminución de las conductas internalizantes, externalizantes y los problemas de comportamiento de los niños y niñas (Mcwey y Cui, 2017).

Este panorama evidencia que el proceso de restablecimiento de derechos no ha sido construido desde una perspectiva familiar y, como consecuencia, no da prioridad al derecho de los niños a ser parte de una familia. Las medidas de restablecimiento de derechos no se orientan a fortalecer y desarrollar las capacidades de las familias para que sean entornos efectivos de protección y cuidado; detrás de las prácticas institucionales, que paradójicamente debilitan el entorno familiar, hay una representación social que descalifica la capacidad de las familias en situaciones de crisis y carencia para hacerse cargo del cuidado y protección de los niños.

Las políticas públicas de protección social deben orientarse sin lugar a dudas a la prevención de todas las formas de maltrato, al acompañamiento de las familias en condiciones de vulnerabilidad y a garantizar, en cuanto sea posible, que los niños y niñas permanezcan bajo el cuidado familiar. En los casos en los cuales el cuidado institucional resulta inevitable es fundamental favorecer dinámicas de acogida y acompañamiento que permitan a los niños establecer vínculos cercanos con sus cuidadores y compañeros, en grupos pequeños en los que se favorezca la autonomía y la autorregulación, de acuerdo con la etapa de desarrollo en la que los niños se encuentran. Asimismo, es muy importante que los cuidadores puedan reconocer su incidencia en la regulación interna que establecen los niños y puedan proponer alternativas que favorezcan la inclusión y minimicen la exclusión.
Algunas instituciones de protección han buscado transitar hacia modelos familiares de acogida, en los que se prioriza el favorecer la ubicación conjunta de grupos de hermanos con cuidadores estables y en espacios físicos no institucionales (apartamentos o casas) similares a los que podrían ser habitados por una familia con lazos biológicos. No obstante, estas modalidades de cuidado siguen siendo excepcionales y no existen lineamientos oficiales que propugnen por la transición hacia alternativas similares que resulten más favorables para el desarrollo psicoafectivo de los niños y adolescentes que necesitan estos espacios de protección.

Por otra parte, es fundamental que los niños puedan elaborar la experiencia de lo vivido y dar al cuidado institucional un sentido protector. Resignificar las propias experiencias para cuestionar las atribuciones causales que remiten únicamente al propio comportamiento puede favorecer dinámicas de resiliencia que incidan en el futuro en un mayor ajuste social y en la disminución del sufrimiento percibido.

La opción del cuidado institucional debe ser cuidadosamente considerada en los casos en donde es pasible el reintegro al grupo familiar de origen. Cuando una institución asume la responsabilidad de la familia puede ratificar en ella la percepción de su incapacidad para el cuidado y la protección de los niños. Por otra parte, el que los niños permanezcan la mayor parte del tiempo en un entorno distinto al familiar dificulta la incorporación en unas dinámicas familiares que resultan ajenas y esta conflictividad reafirma a su vez el sentido de ineficacia que experimenta la familia en el contener y orientar a los niños y niñas. Además, el hecho de que en el entorno institucional existan figuras de afecto y autoridad que se sobreponen y ejercen poder sobre los adultos significativos de la familia de origen comunica a los niños y niñas un sentido de inseguridad frente a las posibilidades de estos para proveer apoyo efectivo y para fungir como referentes de autoridad (Minuchin et al., 2000).

A esto se suma la carencia económica de muchas de estas familias que genera un fuerte contraste entre las condiciones de vida a las que acceden los niños dentro de la institución y las que enfrentan con sus familias de origen. 
Una intervención con perspectiva familiar debería orientarse a priorizar el fortalecimiento de la familia, la identificación de recursos y posibles redes de apoyo y buscaría minimizar el tiempo de permanencia en el entorno institucional, pues cuanto más dura la separación, más difícil resulta la reunificación (Ordóñez, 2009; Villegas y Pecora, 2012). Los vínculos que generan la pertenencia a un núcleo familiar se tejen en el tiempo y la cotidianidad, en el compartir espontáneo y en las dinámicas que cada familia construye como parte de su identidad. Se trata de una experiencia de vinculación que puede favorecerse y fortalecerse a partir de intervenciones puntuales, pero que no se improvisa en ellas, porque requiere de la autonomía de los sujetos que interactúan y se narran a sí mismos a partir de las interacciones que establecen con otros.

Cuando es inevitable la acogida institucional, el tipo de relación que se establece entre la familia y la institución es determinante para la posibilidad exitosa de un reintegro a corto plazo. Cuando la institución promueve, estimula y facilita las visitas y el contacto frecuente de los niños con su familia de origen se incrementan las probabilidades de una reunificación familiar exitosa; además, esta es una variable relacionada con el bienestar infantil en contextos institucionales, en términos de seguridad, ajuste emocional, social y escolar (Proch y Howard, 1986).

El trabajo con las madres sustitutas y con los profesionales que acompañan el proceso resulta fundamental, en tanto se posibilite una nueva mirada sobre las familias de origen que se oriente a la búsqueda y movilización de recursos y no a la estigmatización sobre las carencias, que permita el desarrollo de la autonomía y la autoeficacia confiriendo poder a la familia biológica.

A pesar de los avances en los procesos de protección a la infancia, continuamos en deuda de generar alternativas de intervención que permitan a los niños y niñas que han vivido experiencias de ruptura, maltrato y abandono la posibilidad de reconstruir "la casa", de manera que puedan vincularse con otros en la regeneración de un tejido social que no continúe perpetuando los ciclos de violencia y que sea capaz de acoger y cuidar a los más pequeños.

\section{Referencias}

Bowlby, J. (2012). A Secure Base: Clinical Applications of Attachment Theory. Nueva York: Routledge.

Benavidez, J. y Miranda, S. (2007). Actitud crítica hacia el castigo físico en niños víctimas de maltrato infantil. Universitas Psychologica, 6(2), 309-318.

Recuperado de https://revistas.javeriana.edu.co/ index.php/revPsycho/article/view/119

Carreño, C. (2017). Las madres, las familias y los hijos del sur: Miradas etnográficas a la protección infantil contemporánea en la ciudad de Bogotá (tesis de Doctorado en Antropología). Universidad Nacional de Colombia, Bogotá.

De La Iglesia, M. y Di lorio, J. (2006). La infancia institucionalizada: la práctica de la Psicología Jurídica. Determinantes institucionales. Anuario de investigación. Recuperado de http://www.scielo.org.ar/ scielo.php?pid=S1851-16862006000100032\&script=sci_arttext

Durán, E. y Valoyes, E. (2009). Perfil de los niños, niñas y adolescentes sin cuidado parental en Colombia. Revista Latinoamericana de Ciencias Sociales, Niñez y Juventud, 7(2). Recuperado de http://revistaumanizales.cinde.org.co/rlcsnj/index.php/Revista-Latinoamericana/article/ view/192

Gómez, E. y Haz, A. M. (2008). Intervención familiar preventiva en programas colaboradores del Sename: la perspectiva del profesional. Psykhe, 17(2), 53-65. https://doi.org/10.4067/ s0718-22282008000200005

González, M. J. (2014). Mental health care of families affected by the child welfare system. Child Welfare, 93(1), 7-58. Recuperado de https://www.ncbi.nlm.nih.gov/pmc/articles/ PMC2857415/

Gypen, L., Vanderfaeillie, J., Maeyer, S. De, Belenger, L. y Holen, F. Van. (2017). Outcomes of children who grew up in foster care: Systematic-review. Children and Youth Services Review, 76, 74-83. https://doi.org/10.1016/j. childyouth.2017.02.035

Hirschfeld, L. (2002). Why don't anthropologists like children? American Anthropologist, 104(2), 611-627. https://doi.org/10.1525/ aа.2002.104.2.611 
McWey, L. M. y Cui, M. (2017). Parent-child contact for youth in foster care: Research to inform practice. Family Relations, 1491, 684-695. https://doi.org/10.1111/fare.12276

Minuchin, P., Colapinto, J. y Minuchin, S. (2000). Pobreza, institución, familia. Buenos Aires: Amorrortu.

Nadeem, E., Waterman, J., Foster, J., Paczkowski, E., Belin, T. R. y Miranda, J. (2017). Long-Term effects of pre-placement risk factors on children's psychological symptoms and parenting stress among families. Journal of Emotional and Behavioral Disorders, 25(2), 67-81. https://doi. org/10.1177/1063426615621050

Naumova, O. Y., Rychkov, S. Y., Kornilov, S. A., Odintsova, V. V., Anikina, V. O., Solodunova, M. Y., Arintcina, I. A., Zhukova, M. A., Ovchinnikova, I. V., Burenkova, O. V., Zhukova, O. V., Muhamedrahimov, R. J., Grigorenko, E. L. (2019). Effects of early social deprivation on epigenetic statuses and adaptive behavior of young children : A study based on a cohort of institutionalized infants and toddlers. PlosOne, 1-30. https://doi.org/10.1371/journal. pone.0214285

Ordóñez, M. D. (2009). Factores que influyen en el éxito de la reunificación familiar. Revista Internacional de Ciencias Sociales y Humanidades, 19(1), 139-161. Recuperado de https://www.redalyc.org/articulo.oa?id=65415751007 https:// doi.org/10.29057/icshu.v1i1.882

Perego, G., Caputi, M. y Ogliari, A. (2016). Neurobiological correlates of psychosocial deprivation in children: A systematic review of neuroscientific. Child Youth Care Forum, 45, 329-352. https://doi.org/10.1007/s10566-015-9340-z

Proch, K. y Howard, J. A. (1986). Parental visiting of children in foster care. Social Work, 31(3), 178-181. https://doi.org/10.1093/sw/31.3.178

Sherr, L., Roberts, K. J. y Gandhi, N. (2017). Child violence experiences in institutionalised/orphanage care. Psychology, Health \& Medicine, 22(51), 31-57. https://doi.org/10.1080/135485 06.2016 .1271951

Touza, C. (2002). Las consecuencias del maltrato y del abandono en el desarrollo socioemocional de niños y adolescentes institucionalizados (Tesis Doctoral). Recuperado de http://eprints. ucm.es/3028/

Troller-Renfree, S., Nelson, C. A., Zeanah, C. H. y Fox, N. A. (2016). Deficits in error monitoring are associated with externalizing but not internalizing behaviors among children with a history of institutionalization. The Journal of Child Psychology and Psychiatry, 10(57), 1145-1153.

Villalta, C. (2013). Un campo de investigación. Las técnicas de gestión y los dispositivos jurídico-burocráticos destinados a la infancia pobre en Argentina. Civitas, 13(2), 245-268. https:// doi.org/10.15448/1984-7289.2013.2.15482

Villegas, S. y Pecora, P. J. (2012). Mental health outcomes for adults in family foster care as children : An analysis by ethnicity. Children and Youth Services Review, 34(8), 1448-1458. https://doi. org/10.1016/j.childyouth.2012.03.023 